\title{
High Peak Power, Single-polarized, Sub-nanoseconds Pulses Generation of a Yb-doped Rod-type Photonic Crystal Fiber Amplifier"
}

\author{
Ziwei Wang ${ }^{1,2}$, Songtao Du ${ }^{1}$, Zhaokun Wang ${ }^{1,2}$, Jing $\mathrm{He}^{1,2}$, Yunrong Wei ${ }^{1}$, Qihong Lou ${ }^{1}$, Jun Zhou ${ }^{1}$ \\ ${ }^{1}$ Shanghai Key Laboratory of All Solid-State Lasers and Applied Techniques, Shanghai Institute of Optics and Fine Mechanics, \\ Chinese Academy of Sciences, Shanghai, China \\ ${ }^{2}$ University of Chinese Academy of Sciences, Beijing, China
}

Received 2013

\begin{abstract}
We report a high-peak-power, single-polarized master oscillator power amplification system employing polarizationmaintaining Yb-doped rod-type photonic crystal fiber. The MOPA system comprises of a Q-switched microchip laser generating $\sim 630$ ps pulses at $8.6 \mathrm{kHz}$ repetition-rate and two amplification stages employing double cladding fiber and rod-type PCF respectively. The MOPA system obtains narrow spectral bandwidth, single-polarized pulses of 9W maximum output average power, corresponding to peak power of 1.7MW.
\end{abstract}

Keywords: Lasers; MOPA; Rod-type PCF; Single Polirization; High-peak-power

\section{Introduction}

High-peak-power, single-polarized laser sources operating in sub-nanosecond regime are widely used in a range of applications including frequency conversion [1], material processing [2] and remote sensing[3]. Rare-earthdoped fiber lasers and amplifiers with single-polarization, narrow spectral linewidth, high signal-to-noise ratio and excellent beam quality offer manifest advantages in the applications above. In the past few years, large mode area (LMA) double cladding fibers (DCFs) are widely used in short-pulsed fiber amplifiers. In 2005, Fabio Di Teodoro et al. [4] reported a three-stage amplifier generating pulses whose peak power in excess of $4.5 \mathrm{MW}$ while pulse duration was 450ps and repetition rateswas $13.4 \mathrm{kHz}$. In 2010, Shaif-ul Alam et al. [5] introduced a single-polarized master oscillator power amplification (MOPA) system with output peak power of $39 \mathrm{~kW}$ at 20ps pulse duration $113.8 \mathrm{MHz}$ repetition rate. Although LMA DCF can support high power for pulsed lasers, it would become multiple transverse modes with beam quality reduction [6]; moreover, there isn't polarization-maintaining (PM) LMA DCF whose core diameter is larger than $30 \mu \mathrm{m}$ in the market. In 2007, a $\sim 85 \mathrm{ps}$ pulse duration, $105 \mathrm{kHz}$ repetition-rate, 3MW peak power Yb-doped fiber amplifier exhibiting narrow spectrum and diffraction-limited beam quality was reported by $\mathrm{O}$.

*The work has been sponsored by the national foundation of China ( NO.60908011, NO.60907045)
Schmidt et al. [7]. The significant component in the amplifier was a very-large-core, intrinsically single-mode, rod-type photonic crystal fiber (PCF) [8] in power amplification stage. The rod-type fiber combines advantages of fiber lasers and bulk solid lasers by particular microstructure design. Through enlarging core diameter and reducing length, the peak power in core is lower. Furthermore, it is designed as an extraordinary small refractive-index difference between core and cladding to be intrinsically single mode. Although the rod- type fiber cannot be coiled as ordinary fibers, it presents a range of eminent features including single-mode operation, pump acceptance angle and efficient pump absorption. The paper discussed above reported a narrow pulse, high-peak-power results, however, the damage of main amplifier was observed at maximum power and pulse distortion was also occurred when peak power was $\sim 2 \mathrm{MW}$. The papers about rod-type PCF amplifier are mainly focused on non-polarized amplification so far, nevertheless high-peak-power PM amplifier is rarely reported.

In this paper, we present a single-polarized dual-stage master oscillator power amplification (MOPA) system for generating high-peak-power and single-polarized pulses. We employ a low-repetition-rate passively Q-switched microchip laser as seed, and introduce Yb-doped, PM, LMA double cladding fiber and 80cm-long, 100 $\mathrm{m}$-core, $\mathrm{PM}$, rod-type PCF as gain medium in pre-amplification and power amplification stage respectively. From this system, we obtained $\sim 600 \mathrm{ps}, 8.6 \mathrm{kHz}$ repetition-rate, center 
wavelength $1064 \mathrm{~nm}$, and spectrum linewidth $0.46 \mathrm{~nm}$, polarization extinction ratio (PER) 13dB laser output of average power $9 \mathrm{~W}$, corresponding to a peak power exceeding 1.7MW and pulse energy exceeding $1 \mathrm{~mJ}$.

\section{Experimental Setup}

The experimental architecture is shown in Figure 1. A fiber-pigtailed $\mathrm{Nd}: \mathrm{YAG} / \mathrm{Cr}^{4+}: \mathrm{YAG}$, single-polarized, passively Q-switched microchip laser is used as the master oscillator(MO) coupling into amplifier. Due to the low gain of short Yb-doped fibers in 1064nm, the MO output is amplified in a dual-stage, PM fiber amplifier chain that affords effective suppression of nonlinearities such as amplified spontaneous emission(ASE) and simulated Raman scattering(SRS). Polarization independent optical isolators are used between amplification stages to prevent backward laser leakage. Cladding power stripper is also introduced to protect $\mathrm{MO}$ against backward pump laser. For the first amplification stage, we use a 3m-long PM Yb-doped double cladding fiber(core/cladding diameter $25 / 250 \mu \mathrm{m}$, core/cladding NA 0.06/0.46 and pump absorption $\sim 5.10 \mathrm{~dB} / \mathrm{m} @ 975 \mathrm{~nm}$ ) as gain medium. The fiber is backward pumped by two fiber-pigtailed diode lasers at $975 \mathrm{~nm}$ through a $(2+1) \times 1$ combiner whose end is spliced with endcap and angle $\left(6^{\circ}\right)$ polished to prevent facet damage and backward-reflected power from coupling into fiber core. We employ two half-wave plates to control the polarization of pre-amplified output pulses. To remove all remaining ASE and preventing backward pump light of the second stage, an inter-stage band-pass filter is specialized designed, emitting only a spectral band of $10 \mathrm{~nm}$ centered at $1064 \mathrm{~nm}$. The filtered pre-amplified pulses are coupled into a 80cm-long, PM Ybdoped rod-type PCF (core/cladding diameter 100/285 $\mu \mathrm{m}$, core/cladding NA 0.02/0.6, and pump absorption $\sim 30 \mathrm{~dB} /$ m@975nm) which is free-space backward pumped by a $\mathrm{LD}$ at $975 \mathrm{~nm}$ through a set of lens and dichroic mirrors (1064nm HT\&975nm HR). The rod-type PCF is endcapping with large AR-coating silica columns(length $8 \mathrm{~mm}$ and diameter $8.2 \mathrm{~mm}$ ) in both ends in order to provide appropriate beam expansion, thus reduce end facet intensity and avoid damage of end facet for a $0^{\circ}$ angle facet. The amplified beam outputs through a collimating lens.

The output characterization of the fiber amplifier includes average power, spectrum and temporal pulse profile. We measure average power by Thorlabs S370C power meter. Spectrum and temporal profile are detected by Yokogawa AQ6370 optical spectrum analyzer and LeCroy Wavesurfer 104MXs $1 \mathrm{GHz}$ oscilloscope connected to a high speed InGaAs photodiode respectively.

\section{Results and Discussion}

The spectrum (a), pulse temporal profile (b) of MO are presented in Figure 2. The average output power of MO is $\sim 170 \mathrm{~mW}$ with center wavelength at $1064.8 \mathrm{~nm}, 3 \mathrm{~dB}$ spectral bandwidth of $0.29 \mathrm{~nm}$, pulse duration of $628 \mathrm{ps}$ and $8.6 \mathrm{kHz}$ repetition-rate. And the PER of the MO is $\sim 13 \mathrm{~dB}$.

Figure 3 provides the average output power of the MOPA system as the function of launched pump power. After pre-amplification, there is average power $\sim 500 \mathrm{~mW}$ filtered pulses coupled into rod-type PCF. The maximum output average power is $9 \mathrm{~W}$ corresponding to peak power exceeding $1.7 \mathrm{MW}$, when pump power is $31 \mathrm{~W}$. The slope efficiency extracted form a linear fit is $\sim 44 \%$. It is emerged that signal power increases along with pump power increase; the pump threshold of rod-type fiber is $\sim 5 \mathrm{~W}$. Moreover, the shift in wavelength of pump

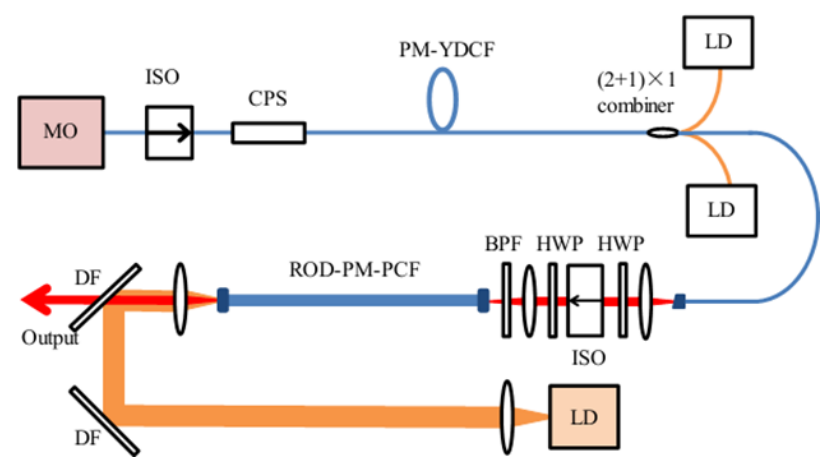

Figure 1. experimental setup. MO: master-oscillator; ISO: optical isolator; CPS: cladding power stripper; PM-YDCF: polarization-maintaining Yb-doped double cladding fiber; LD: laser diode; HWP: half-wave plate; BPF: band-pass filter; ROD-PM-PCF: rod-type polarization-maintaining photonic crystal fiber; DF: dichroic mirror.
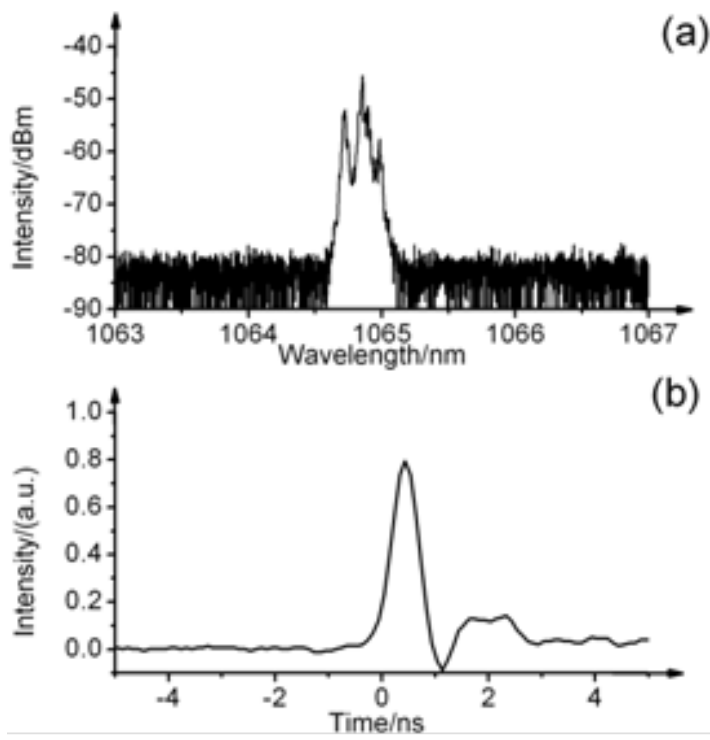

Figure 2. Seed's features of (a) spectrum and (b) temporal profile. 
would move towards the Yb-absorption peak at 975nm with pump power increasing. Owing to the endcaps, the maximum in-core peak intensity of $68 \mathrm{GW} / \mathrm{cm}^{2}$ is reduced to $\sim 10 \mathrm{MW} / \mathrm{cm}^{2}$, which is far less than the measured surface damage threshold for sub-nanosecond pulses in silica [9]. We suggest that significant higher amplified signal power scaling would be possible through increasing pump power as power saturation or deduction isn't occurred. Through increasing the power of pre-amplified pulses, both the output power and optical-to-optical efficiency would increase notably . Furthermore, we measure the PER to be $\sim 13 \mathrm{~dB}$ at maximum output operation, that means the amplifier maintaining the polarization of MO.

In Figure 4, the typical spectrum of seed pulses (a) is presented comparing with output pulses of average power at $9 \mathrm{~W}$ (b). The output center wavelength is $1064.9 \mathrm{~nm}$, and $3 \mathrm{~dB}$ spectral bandwidth is $0.46 \mathrm{~nm}$. The amplified spectrum is relatively broader than seed's, it is due to self-phase modulation (SPM) [10] in high peak power. Thanks to the effective management of continuous wave ASE, the signal to peak $(\sim 1040 \mathrm{~nm})$ ASE ratio exceeds

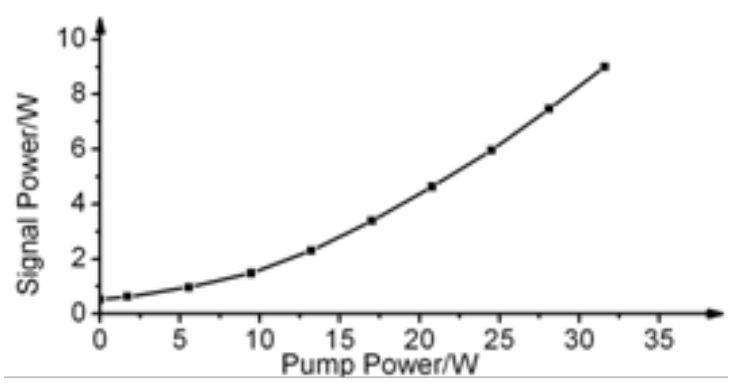

Figure 3. Average output power of signal laser versus pump power.
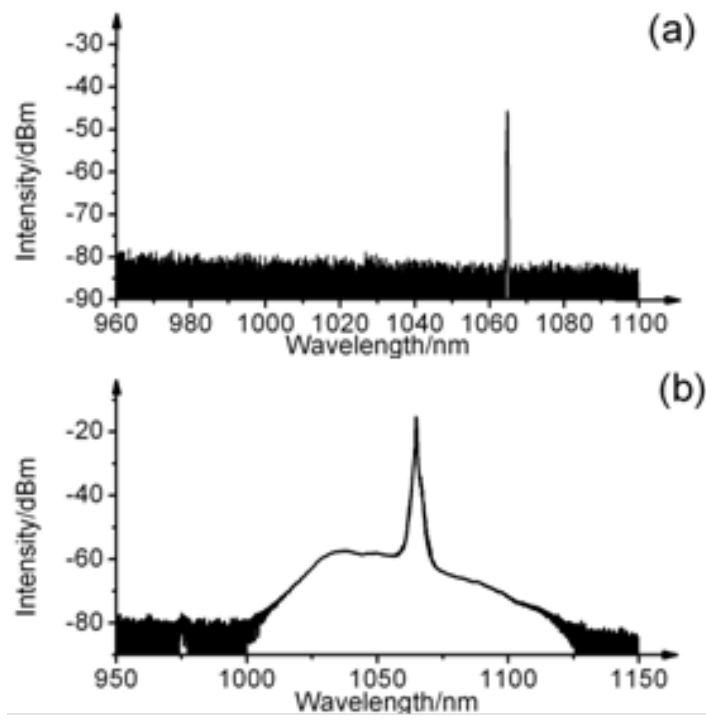

Figure 4. (a) seed spectrum and (b) amplified spectrum at output power of $9 \mathrm{~W}$.
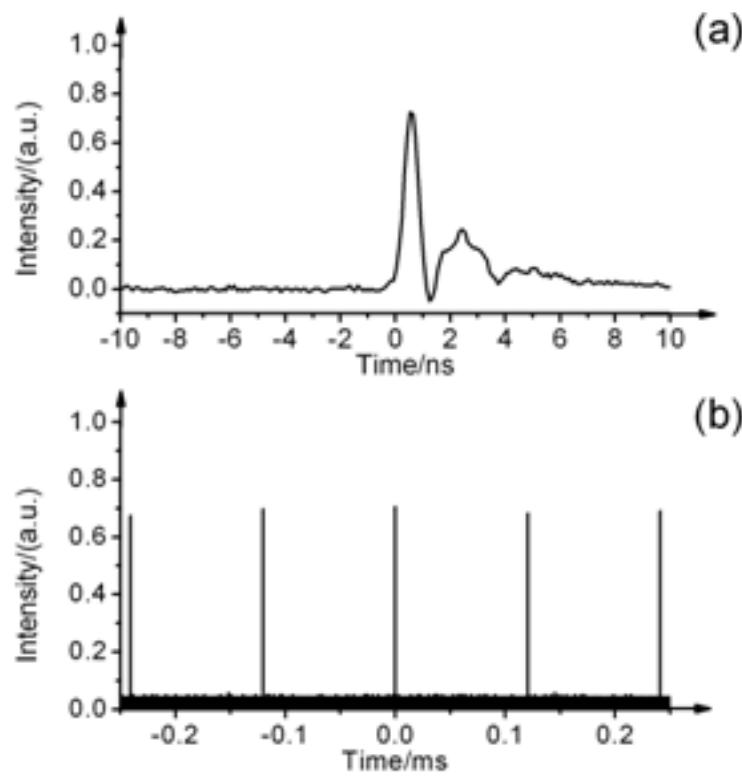

Figure 5. Amplified pulse (a) temporal profile and (b) sequence at output power of $9 \mathrm{~W}$.

40dB; SRS and four-wave-mixing (FWM) are not engendered in the measurement. Furthermore, because of backward pump, residual pump is hardly appeared. In general, the spectrum represents an excellent signal-tonoise ratio of the system, and it demonstrates the benefits of rod-type PCFs in minimizing nonlinear effects.

Figure 5 shows the temporal profile (a) and pulse sequence (b) of output pulses at maximum average power. The output pulse duration is 632ps while repetition rate is $8.6 \mathrm{kHz}$. Comparing to the seed pulse profile, an irregular temporal pulse distortion is exhibited owing to gain saturation and SPM corresponding to the spectrum broadening we discussed above. SPM would become significant constraint in further power amplification scaling.

\section{Conclusions}

In this paper, we report on a single-polarized, dual-stage MOPA system combining Q-switched microchip laser with Yb-doped very-large-mode-area rod-type PCF in sub-nanosecond regime. The amplifier generates high peak power in excess of 1.7MW (corresponding to average power $9 \mathrm{~W}$, pulse duration 638ps and repetition rate $8.6 \mathrm{kHz}$ ) pulses in single-polarized, spectrally narrow, high signal-to-noise ratio beam, which haven't been generated in Yb-doped double cladding fiber amplifiers. This work represents the highest peak power obtained from single-polarized rod-type PCF amplifiers. With the excellent properties including high-peak-power and single-polarization, the MOPA system is a promising candidate to be used in applications including frequency conversion, material processing and LIDAR. In the future work, we would donate attempts to increase pulse 
energy before main amplification and improve the polarization of the system while maintains the simplicity and stability of the system.

\section{REFERENCES}

[1] J. Saby, B. Cocquelin, A. Meunier, S. Pierrot, P.-J. Devilder, P. Deslandes and F. Salin, "High Average and High Peak Power Pulsed Fiber Lasers at 1030 nm, 515nm and 343 nm,” Proceedings of SPIE, Vol. 7580.

[2] O. Schmidt, J. Rothhardt, F. Röser, S. Linke, T. Schreiber, K. Radem aker, J. Limpert, S. Ermeneux, P. Yvernault, F. Salin and A. Tünnermann, "Millijoule Pulse Energy Q -Switched Short-Length Fiber Laser," Optics Letters, Vol.32, 2007, pp. 1551-1553.

[3] J. Dong, A. Shirakawa1, S. Huang, Y. Feng1, K. Takaichi, M. Musha, Ken-Ichi Ueda and A. A. Kaminskii, "Stable Laser-Diode Pumped Microchip Sub-Nanosecond Cr, Yb: YAG self-Q-switched laser," Laser Physics Letters, Russia, Vol. 2, 2005,pp. 387-391. doi:10.1002/lapl.200510018

[4] F. D. Teodoro and C. D. Brooks, "Multistage Yb-Doped Fiber Amplifier Generating Megawatt Peak-Power, Subnanosecond Pulses," Optics Letters, Vol. 30, 2005, pp. 3299-3301.

[5] S.-U. Alam, K.K. Chen, J. R. Hayes, D. J. Lin, A. Mali- nowsky, H.J. Baker, N. Trela, R. McBride and D. J. Richardson, "Over 55W of Frequency Doubled Light at $530 \mathrm{~nm}$ Pumped by an All-Fiber, Diffraction Limited, Picosecond Fibre MOPA,” Proceedings of SPIE, Vol. 7580 .

[6] J. Limpert, F. Röser, D. N. Schimpf, E. Seise, T. Eidam, S. Hädrich, J. Rothhardt, C. J. Misas and A. Tünnermann, "High Repetition Rate Gigawatt Peak Power Fiber Laser Systems: Challenges, Design, and Experiment," IEEE Journal of Selected Topics in Quantum Electronics, Vol. 15, 2009, pp.159-169. doi:10.1109/JSTQE.2008.2010244

[7] O. Schmidt, D. Nodop, J. Limpert, A. Tünnermann, “105 kHz, 85 ps, 3 MW Peak Power Microchip Laser Fiber Amplifier System,” OSA Technical Digest Series (CD) (Optical Society of America, 2008), paper WB23.

[8] F. D. Teodoro and C. D. Brooks, "Fiber Lasers: Fiber Sources Reach Multimegawatt Peak Power in NS Pulses", Laser Focus World, 2006, pp. 94-98.

[9] D. Taverner, D. J. Richardson, L. Dong and J. E. Caplen, "158-mJ Pulses from A Single-Transverse-Mode, LargeMode-Area Erbium-doped fiber amplifier,” Optics Letters, Vol.22, 1997, pp. 378-380.

[10] G. P. Agrawal, Nonlinear Fiber Optics, 4th. Edition. Oxford: Elsevier, 2007. 